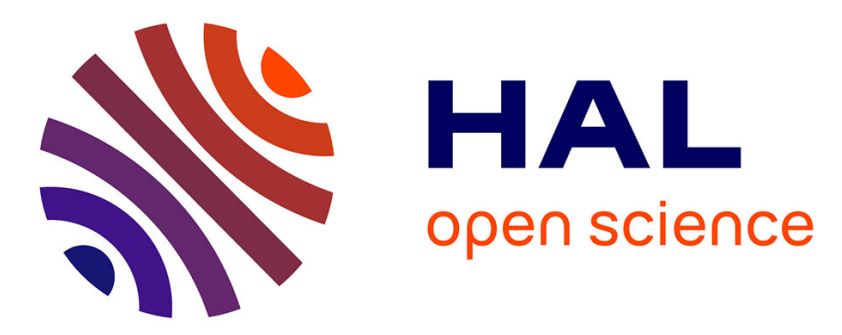

\title{
Quelle est la nature des problèmes posés en visualisation par écrans plats matriciels à cristaux liquides?
}

\author{
G. Labrunie
}

\section{To cite this version:}

G. Labrunie. Quelle est la nature des problèmes posés en visualisation par écrans plats matriciels à cristaux liquides?. Revue de Physique Appliquée, 1984, 19 (2), pp.107-108. 10.1051/rphysap:01984001902010700 . jpa-00245159

HAL Id: jpa-00245159

https://hal.science/jpa-00245159

Submitted on 1 Jan 1984

HAL is a multi-disciplinary open access archive for the deposit and dissemination of scientific research documents, whether they are published or not. The documents may come from teaching and research institutions in France or abroad, or from public or private research centers.
L'archive ouverte pluridisciplinaire HAL, est destinée au dépôt et à la diffusion de documents scientifiques de niveau recherche, publiés ou non, émanant des établissements d'enseignement et de recherche français ou étrangers, des laboratoires publics ou privés. 


\title{
Quelle est la nature des problèmes posés en visualisation par écrans plats matriciels à cristaux liquides?
}

\author{
G. Labrunie \\ LETI/CES, Commissariat à l'Energie Atomique, CEN-G, 85X, 38041 Grenoble Cedex, France
}

(Reçu le 3 juin 1983, accepté le ler septembre 1983)

\begin{abstract}
Résumé. - On définit les caractéristiques principales qui doivent faire l'objet d'améliorations dans les nouvelles générations d'écrans plats à cristaux liquides. On en déduit, suivant les diverses structures d'écrans, les sujets d'étude correspondants et le sens des efforts de $\mathrm{R}$ et $\mathrm{D}$ qui restent à accomplir.
\end{abstract}

\begin{abstract}
Main characteristics which must be enhanced for new generations of liquid crystal flat panels are defined. The corresponding research topics are deduced, depending on the various panels structures, together with the direction of $\mathbf{R}$ and $\mathbf{D}$ efforts remaining to be pursued.
\end{abstract}

\section{Introduction.}

Le domaine de la visualisation par écrans plats subit actuellement une évolution très rapide dont une des caractéristiques principales est le passage des afficheurs dédiés aux écrans matriciels. Cette évolution s'accompagne d'une amélioration de :

- La définition, vers la haute complexité $\left(10^{5}\right.$ $10^{6}$ points).

- La surface, vers l'écran mural $\left(1 \mathrm{dm}^{2}\right.$ à $\left.1 \mathrm{~m}^{2}\right)$.

- L'aspect, caractérisé par un meilleur contraste et l'apparition d'écrans trichromes.

- L'interactivité, avec comme objectif la compatibilité avec les sources de données fonctionnant à la cadence vidéo.

- La consommation, de façon à ce que l'écran puisse fonctionner sur batteries (puissance $<1 \mathrm{~W}$ ).

Cependant, cette évolution doit rester compatible avec des domaines d'application aussi variés que possible, et l'écran doit donc faire appel à des circuits intégrés de technologie standard pour la commande, à des procédés d'interconnexion simples et peu coûteux, et il doit fonctionner correctement dans des conditions sévères d'environnements. $\mathrm{Ce}$ papier résume les problèmes à résoudre pour atteindre ces objectifs avec diverses techniques d'écrans plats matriciels à cristaux liquides.

\section{Les structures d'écrans.}

On distingue actuellement deux grandes options techniques dans ce domaine :

- Les écrans à multiplexage direct, constitués simplement d'un double réseau d'électrodes transparentes déposées sur verre, entre lesquelles est introduite la couche active de cristal liquide. Cette technologie "simple" conduit à faire porter l'essentiel de l'effort technique sur la chimie du cristal liquide, comme nous le verrons, ainsi que sur la technologie hybride d'interconnexion des C.I. de commande.

- Les écrans à commande intégrée (ou « matrice active "), dans lesquels le cristal liquide est compris entre une électrode transparente uniforme et un double réseau coplanaire de bus électriques adressant un transistor en série avec chaque point image. Cette technique de circuit intégré grande surface (TMOS sur Si monocristallin ou TCM - transistor en couche mince - sur support transparent) permet en principe d'inclure la logique et les registres de commande autour de la zone active, déplaçant ainsi l'essentiel des problèmes de la physico-chimie du cristal liquide vers la physique et la technologie de ce circuit intégré.

2.1 ECRANS À MUltipleXaGe DireCt. - On ne traite ici que des effets non bistables, donc essentiellement 
nématique en hélice (N.H.) et biréfringence contrôlée électriquement (B.C.E.).

L'analyse détaillée des cinq critères d'évolution énoncés précédemment permet de mettre en évidence dans chaque cas l'origine des limitations constatées : le taux de multiplexage pour le nombre de lignes, le contraste et l'angle de vue pour l'aspect, la fréquence image pour l'interactivité, la tension et le courant de commande pour la consommation.

Après remise en ordre des problèmes posés (nature, importance) on est conduit à définir comme suit les grands axes d'étude qui apparaissent ici.

- Chimie des cristaux liquides (C.L.) :

2, 3)

- améliorations des constantes élastiques $k_{i i}(i=1$,

- adaptation de la biréfringence $\Delta n$ à l'épaisseur $e$

- accroissement de $|\Delta \varepsilon|$

- réduction de la viscosité

- extension de la gamme de mésomorphisme.

- Technologie de la cellule :

- physico-chimie des traitements d'orientation

- réduction de l'épaisseur $e$ et de la tolérance $\Delta e$

- amélioration de la conductivité des électrodes transparentes en ITO.

2.2 ECRANS INTÉGRÉs. - La même méthode d'analyse fait apparaître des problèmes de natures beaucoup plus diverses. Ceci est dû en particulier au fait que si les limitations de l'aspect, de l'interactivité et de la consommation ont à peu près les mêmes origines que précédemment, le nombre de lignes est essentiellement limité par les propriétés physiques du semiconducteur employé, et la surface utile par la taille de la matrice intégrée réalisable.

On est ainsi conduit à distinguer dans ce domaine beaucoup plus neuf que le précédent quatre directions principales d'étude.

- Physique du semiconducteur : méthode de dépôt, caractérisation électronique, dopage, stabilité, recristallisation.

- Physique du composant T.C.M. : structure, nature de l'isolant, caractéristiques électriques (valeurs du courant dans chaque état, bande passante), uniformité.

- Amélioration des rendements de fabrication par introduction de certaines formes de redondance dans la conception de la matrice.

- Chimie des C.L. : mise au point de mélanges dichroïques de haute efficacité :

- Base cholestérique/nématique : amélioration de l'anisotropie optique $(\Delta n)$ et diélectrique $(\Delta \varepsilon)$, de la gamme de mésomorphisme, et réduction de la viscosité.

- Colorant dichroïque : amélioration du rapport dichroïque, de la solubilité dans la base et de la stabilité aux U.V.

\section{Conclusion.}

Que peut-on espérer de ces techniques d'écrans matriciels à cristaux liquides? L'évolution actuelle des écrans à multiplexage direct vers les écrans intégrés a pour origine le souci de séparer la fonction « multiplexage » de la qualité visuelle de l'écran. Elle permet de relaxer les contraintes imposées au C.L. et de trouver des solutions indépendantes, donc d'améliorer simultanément l'ensemble des caractéristiques des écrans.

Il est clair cependant que cette intégration se fait aux dépens de la technologie, et donc du coût de fabrication de la cellule.

Si l'on considère maintenant le coût de la fabrication de l'écran complet, incluant ses circuits d'interface, de logique et de commande, il est vraisemblable que les écrans intégrés constitueront à terme une solution dont le rapport performances/prix sera très compétitif. 